\title{
Role of an MDM4 polymorphism in the early age of onset of nasopharyngeal carcinoma
}

\author{
YAO WEI ZHANG ${ }^{1}$, JIAN GUAN ${ }^{1}$, YONG ZHANG ${ }^{2}$, YU RONG QIU ${ }^{3}$ and LONG HUA CHEN ${ }^{1}$ \\ ${ }^{1}$ Department of Radiation Oncology, Nanfang Hospital, Southern Medical University, Guangzhou 510515; \\ ${ }^{2}$ Department of Radiation Oncology, the First Affiliated Hospital of Kunming Medical College, Kunming 650031; \\ ${ }^{3}$ Department of Laboratory Medicine, Nanfang Hospital, Southern Medical University, Guangzhou 510515, P.R. China
}

Received December 15, 2011; Accepted February 24, 2012

DOI: $10.3892 / \mathrm{ol} .2012 .630$

\begin{abstract}
Mouse double minute 4 (MDM4) is a critical negative regulator of the tumor suppressor p53. The results of studies have revealed that an MDM4 polymorphism (rs1563828) may contribute to the earlier onset of several malignant diseases. However, the correlation between this polymorphism and nasopharyngeal carcinoma (NPC) susceptibility has not been explored. We performed a case-control study with 210 NPC patients and 200 healthy controls. Significant associations were found when comparing the age of onset of NPC according to the rs1563828 genotype $(\mathrm{P}=0.01)$. The average age of onset of NPC in patients with the TT, CC and CT genotypes was 39.3, 48.2 and 45.5 years, respectively. Homozygous variant (TT) carriers developed NPC at an earlier age than homozygous (CC) carriers, such that the age of onset was accelerated by 8.9 years $(\mathrm{P}=0.002)$. Our data suggest that $\mathrm{rs} 1563828$ is a modifier of the age of onset of NPC in the population studied. The age of onset for NPC with TT homozygotes was earlier than CC carriers.
\end{abstract}

\section{Introduction}

Nasopharyngeal carcinoma (NPC) is a tumor that arises from epithelial cells. NPC occurs with high incidence in Southeast Asia, particularly in Southern China (1). Notably, the incidence of NPC remains high in the Southern Chinese population even after emigration from China. The high prevalence of NPC in the population suggests that genetic factors contribute to increased cancer risk by affecting susceptibility (2).

NPC is mainly associated with genetic factors and Epstein-Barr virus (EBV) infection (1). The etiology of NPC follows a multi-step process in which $\mathrm{EBV}$, genetic factors and

Correspondence to: Dr Long Hua Chen, Department of Radiation Oncology, Nanfang Hospital, Southern Medical University, Guangzhou 510515, P.R. China

E-mail: clhfl@hotmail.com

Key words: case-control, genetic, susceptibility, molecular epidemiology environmental carcinogens all appear to be significant (3). In order to clarify the pathogenesis of this malignancy, it is necessary to identify the susceptibility genes of NPC.

The tumor suppressor p53 and its negative regulator Mouse double minute 2 (MDM2) are crucial in carcinogenesis. The p53 72Arg $>$ Pro and MDM2 309T $>$ G polymorphisms contribute to the risk of NPC $(4,5)$. MDM4, mapped to chromosome 1q32, is a negative regulator of p53 and cooperates with MDM2 to inhibit p53 activity in the cellular response to DNA damage (6). The role of MDM4, a homolog of MDM2, in regulating p53 activity has been identified. The results of previous studies have revealed that MDM4 has a unique role in regulating $\mathrm{p} 53$. Yu et al found that the joint effect of MDM4 variants (rs1380576C $>\mathrm{G}$, rs11801299G $>$ A and $r 10900598 \mathrm{G}>\mathrm{T}$ ) may contribute to the risk of oropharyngeal cancer (7). A single nucleotide polymorphism (SNP) in MDM4 located within intron 10 (designated rs1563828) has been identified and patients with its homozygous variant (TT) were found to develop ER-negative breast cancer at an earlier age than those with the homozygous wild-type (CC) or heterozygous genotypes (8). However, another study found no significant difference in the age of onset of cancer between the different genotypes of rs1563828 (9). These studies, thus far, have not arrived at consistent conclusions. This suggests that MDM4 rs1563828 performs tissue-specific functions. In this study, the association between rs1563828 and NPC was investigated by examining the age of NPC onset based on the rs1563828 genotype.

\section{Materials and methods}

Study subjects. This study included 210 NPC patients and 200 healthy population controls. All subjects were ethnically homogenous Han Chinese. Patients with newly diagnosed NPC were consecutively recruited from September 2008 to December 2010 at the Southern Hospital, Southern Medical University (Guangzhou, China). The patients were from Guangdong Province and its surrounding regions (Southern China) and there were no age, stage or histology restrictions. The tumor node metastasis (TNM) classification of the 2002 American Joint Committee on Cancer was used to determine the tumor staging. Histological type was evaluated according to the World Health Organization (WHO): type 1, keratinizing squamous cell carcinoma; type 2, non-keratinizing squamous 
cell carcinoma; type 3 , undifferentiated carcinoma. The clinical features of the patients are shown in Table I. The control subjects were randomly selected from a database consisting of 1,000 individuals based on a physical examination. The selection criteria included no history of cancer. At recruitment, informed consent was obtained from each subject. This study was approved by the Medical Ethics Committee of Southern Hospital.

AmplificationofMDM4anddirectsequencing.DNAwasisolated using a Qiagen Blood Mini kit (Qiagen, Valencia, CA, USA) from leukocyte cell pellets from the peripheral blood. A 427-bp fragment spanning the MDM4 rs1563828 region was amplified using forward (5'-TGTGGTGGGAATGGGGGAAGGAT-3') and reverse (5'-GCACTGCTTTCCCTGACTCAACAC-3') primers. The reaction mixture contained $100 \mathrm{ng}$ genomic DNA, $0.13 \mathrm{mM}$ of each dNTP, $2.5 \mathrm{ng}$ of each primer, 0.13 units of Prozyme DNA polymerase (Takara Enterprise, Hyogo, Japan) and 10X PCR buffer. The PCR assay was performed in three steps: denaturation at $10 \mathrm{~min}$ at $94^{\circ} \mathrm{C}$; then annealing at 35 cycles of $30 \mathrm{sec}$ at $94^{\circ} \mathrm{C}, 30 \mathrm{sec}$ at $60^{\circ} \mathrm{C}$ and $60 \mathrm{sec}$ at $72^{\circ} \mathrm{C}$; and extension at $5 \mathrm{~min}$ at $72^{\circ} \mathrm{C}$. Aliquots of the PCR product were sequenced with an internal sequencing primer 5'-GCACTGCTTTCCCTGACTCAACAC-3' from the reverse direction. All sequencing analyses were performed twice to confirm the results.

Statistical analysis. Genotypes were tested for the Hardy-Weinberg equilibrium (HWE) using public software (http://www.oege.org/software/hardy-weinberg.html). Chi-square $\left(\chi^{2}\right)$ analysis was used to calculate the odds ratio (OR) and its $95 \%$ confidence interval (CI) as a measure of the association between rs1563828 genotypes and categorical variables (gender, histological type of NPC and TNM stage). One-way ANOVA was used to measure the association between the three genotypes and age of onset of NPC. This analysis was performed with the inclusion of a dichotomous indicator for the covariate and genotypes: homozygous variant (TT) and heterozygous (CT) versus homozygous carrier (CC). Unconditional univariate and multivariate logistic regression analyses were carried out to obtain the crude and adjusted OR and $95 \% \mathrm{CI}$. The multivariate adjustment included age
Table I. Distribution of clinical data of patients and controls included in the study.

\begin{tabular}{lcc}
\hline & Patients & Controls \\
\hline Gender, n & 210 & 200 \\
Female, n (\%) & $60(28.6)$ & $89(44.5)$ \\
Male, n (\%) & $150(71.4)$ & $111(55.5)$ \\
Age, years (mean \pm SD) & $46.2 \pm 10.7$ & $33.9 \pm 13.3$ \\
Histological type, n & 210 & \\
WHO I, n & 4 & \\
WHO II, n & 18 & \\
WHO III, n & 188 & \\
\end{tabular}

WHO I, keratinizing squamous cell carcinoma; WHO II, nonkeratinizing squamous cell carcinoma; WHO III, undifferentiated carcinoma.

and gender. The genotype-specific distributions according to age of disease onset were tested by calculating the one minus survival function plots computed using the Kaplan-Meier method with the log-rank and Breslow tests. Two-sided tests of statistical significance were conducted using the SPSS for Windows software (version 13.0, Chicago, IL, USA) using a $5 \%$ level of significance.

\section{Results}

Genotype distributions. In this study, 210 NPC patients and 200 controls from Southern China were genotyped. The distribution of rs1563828 genotypes was in accordance with the HWE in the controls and patients $(\mathrm{P}>0.05)$. Genotype frequencies for CC, CT and TT were 45.0, 44.0 and $11.0 \%$, respectively, for the controls and 46.7, 43.3 and 10.0\%, respectively, for the NPC patients. Significant associations were found when comparing the age of onset of NPC according to the rs1563828 genotype $(\mathrm{P}=0.01$; Table II). No significant differences were identified in the risk of developing NPC between the $\mathrm{CC}, \mathrm{CT}$ and TT groups $(\mathrm{P}=0.92 ; \mathrm{OR}=0.88,95 \% \mathrm{CI}=0.45-1.70$ for $\mathrm{TT}$ versus $\mathrm{CC}$; $\mathrm{OR}=0.95,95 \% \mathrm{CI}=0.63-1.43$ for $\mathrm{CT}$ versus $\mathrm{CC}$; Table III). In

Table II. Analysis of clinicopathological parameters among NPC patients.

\begin{tabular}{|c|c|c|c|c|}
\hline & $\mathrm{CC}$ & $\mathrm{TC}$ & $\mathrm{TT}$ & P-value \\
\hline Gender, $\mathrm{n}$ & 210 & 0.097 & & \\
\hline Female, $\mathrm{n}$ & 22 & 33 & 5 & \\
\hline Male, $\mathrm{n}$ & 76 & 58 & 16 & \\
\hline Age of onset, years (mean \pm SD) & $48.2 \pm 11.0$ & $45.5 \pm 9.1$ & $39.3 \pm 13.1$ & 0.01 \\
\hline WHO type, $\mathrm{n}$ & 210 & 0.648 & & \\
\hline $\mathrm{I}, \mathrm{n}$ & 1 & 3 & 0 & \\
\hline $\mathrm{II}, \mathrm{n}$ & 10 & 6 & 2 & \\
\hline III, $\mathrm{n}$ & 87 & 82 & 19 & \\
\hline
\end{tabular}

WHO I, keratinizing squamous cell carcinoma; WHO II, non-keratinizing squamous cell carcinoma; WHO III, undifferentiated carcinoma; NPC, nasopharyngeal carcinoma. 
Table III. Risk of NPC associated with MDM4 genotypes.

\begin{tabular}{lcccr}
\hline Genotype & Cases $(\mathrm{n}=210)$ & Controls $(\mathrm{n}=200)$ & OR $(95 \% \mathrm{CI})$ & $\mathrm{OR}^{\mathrm{a}}(95 \% \mathrm{CI})$ \\
\hline $\mathrm{CC}$ & 98 & 90 & 1.00 & 1.00 \\
$\mathrm{CT}$ & 91 & 88 & $0.95(0.63-1.43)$ & $1.29(0.80-2.06)$ \\
TT & 21 & 22 & $0.88(0.45-1.70)$ & $1.34(0.61-2.96)$
\end{tabular}

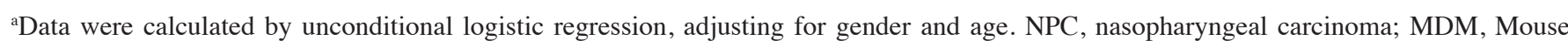
double minute; OR, odds ratio; CI, confidence interval.

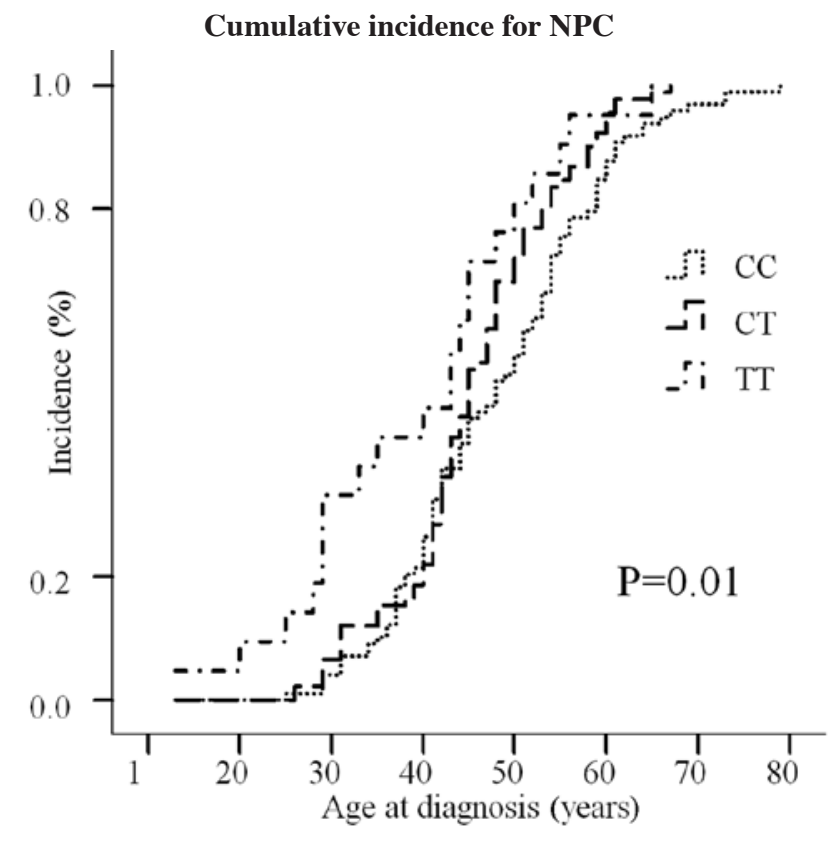

Figure 1. TT genotype of human MDM4 is associated with an accelerated age of onset of NPC in a Southern Chinese population. Kaplan-Meier-estimated cumulative incidence for NPC by MDM4 rs1563828 genotypes (log-rank test, $\mathrm{P}=0.01$ ). The age of onset for TT genotype carriers was earlier than that of CC carriers. MDM, Mouse double minute; NPC, nasopharyngeal carcinoma

the multivariate logistic regression with adjustment for gender and age of onset for NPC, no statistical differences were found $(\mathrm{OR}=1.34,95 \% \mathrm{CI}=0.61-2.96$ for $\mathrm{TT}$ versus $\mathrm{CC} ; \mathrm{OR}=1.29,95 \%$ $\mathrm{CI}=0.80-2.06$ for $\mathrm{CT}$ versus CC; Table III). No significant associations were found by stratifying the NPC cases according to the clinicopathological data (gender and histological type; Table II). The distribution of tumor stage at onset using the TNM classification was not found to be significantly different between the rs1563828 genotypes (data not shown).

Age of onset. We found a statistically significant result when comparing the age of onset of NPC according to the rs1563828 genotype. The results show that homozygous variant (TT) carriers developed NPC at an earlier age than the homozygous (CC) carriers, such that the age of onset was accelerated by 8.9 years $(\mathrm{P}=0.002)$. Moreover, the results of the KaplanMeier analysis estimated the cumulative incidence for NPC by the rs1563828 genotypes. A left shift was observed in the cumulative incidence curve for the TT genotype (log-rank test, $\mathrm{P}=0.01$; Fig. 1).

\section{Discussion}

A large body of evidence suggests that the p53 tumor suppressor pathway is key in reducing cancer frequency in vertebrates. p53 inactivation in cancers may result from the amplification/overexpression of its specific inhibitors MDM2 and MDM4. An SNP in the MDM2 promoter (SNP309) may contribute to the early onset of sporadic and hereditary malignancies in patients with defective p53 (10). MDM4 is one of the key negative regulators of p53 and its overexpression or amplification contributes to carcinogenesis by inhibiting p53 tumor suppressor activity. Upon DNA damage, MDM2dependent degradation of itself and MDM4 contribute significantly to p53 stabilization and activation (11). Certain studies have shown that MDM4 is an essential gene in murine development and that knockout embryos die in utero. This lethal phenotype may be rescued by knocking out the p53 gene. MDM4 is a key modulator of p53 activity in differentiating embryonic stem cells (12). A previous study revealed a novel role for MDM4; in non-tumor cells under stress conditions, MDM4 may act as a positive regulator of p53 activity by controlling p53 levels. The overexpression of MDM4 has been found to significantly increase p53-dependent cell death, in correlation with the enhanced induction of endogenous p53 protein levels (13).

In the present study, rs1563828 appears to be associated with an earlier age of onset of NPC. Furthermore, homozygous variant (TT) carriers develop NPC at an earlier age than homozygous (CC) carriers, such that the age of onset was accelerated by 8.9 years. The rs1563828 polymorphism is located in intron 10. Our study has identified an association between the age of onset of NPC and rs1563828. As the polymorphism may be in linkage disequilibrium with another allele, an alternative locus may be the true functional polymorphism. Previous studies have indicated that there is a major haplotype of the human MDM4 $(14,15)$. This haplotype is tagged by several SNPs, which are in high linkage disequilibrium, across the MDM4 gene, including the polymorphic locus rs1563828. NPC is closely associated with genetic factors, EBV infection and other environmental factors, including tobacco smoking and alcohol consumption. In addition, studies have shown a male preponderance, with a male:female ratio of approximately $3: 1$. In the present study, the ratio was $2.5: 1(16,17)$. We hypothesize that individuals carrying the rs1563828 TT genotype have nasopharyngeal mucosa, which are more susceptible to EBV and carcinogen exposure, resulting in an earlier age of onset of NPC. 
High levels of MDM2 and MDM4 are present in numerous head and neck squamous carcinomas (HNSC). In tumors with wild-type p53, there is often an overexpression of MDM2 or MDM4, which leads to the functional inactivation of p53. However, there is an exception to the rule. For example, MDM4 gene amplification in colorectal cancer cases is rare (18), indicating that the expression of MDM2 or MDM4 may be tissue-specific. However, due to the lack of paraffin-embedded tumor samples, the levels of MDM4 were not examined in this study.

In conclusion, we found that rs 1563828 , located in intron 10 of MDM4, is associated with an earlier age of onset of NPC in a Southern Chinese population. However, to confirm our findings, further molecular studies are required to identify the functionality of this polymorphism.

\section{Acknowledgements}

The authors thank all the individuals who assisted with this study. We would like to give special thanks to Allen, a professor at Harvard University, for her rapid response to our requests for reviews of drafts of this study. This study was supported by grants from the Science and Technology Commission Projects of Guangdong Province (2010B031600245), Natural Science Foundation of Guangdong Province (8451051501001344) and Natural Science Foundation of Guangdong Province (S2011010003915).

\section{References}

1. Wei KR, Yu YL, Yang YY, Ji MF, Yu BH, Liang ZH and Reng X: Epidemiological trends of nasopharyngeal carcinoma in China Asian Pac J Cancer Prev 11: 29-32, 2010.

2. Zhou G, Zhai Y, Cui Y, Zhang X, Dong X, Yang H, He Y, Yao K, Zhang H, Zhi L, et al: MDM2 promoter SNP309 is associated with risk of occurrence and advanced lymph node metastasis of nasopharyngeal carcinoma in Chinese population. Clin Cancer Res 13: 2627-2633, 2007.

3. Li Y, Fu L, Wong AM, Fan YH, Li MX, Bei JX, Jia WH, Zeng YX Chan D, Cheung KM, et al: Identification of genes with allelic imbalance on $6 \mathrm{p}$ associated with nasopharyngeal carcinoma in southern Chinese. PLoS One 6: e14562, 2011

4. Xiao M, Zhang L, Zhu X, Huang J, Jiang H, Hu S and Liu Y: Genetic polymorphisms of MDM2 and TP53 genes are associated with risk of nasopharyngeal carcinoma in a Chinese population. BMC Cancer 10: 147, 2010.

5. Yu H, Huang YJ, Liu Z, Wang LE, Li G, Sturgis EM, Johnson DG and Wei Q: Effects of MDM2 promoter polymorphisms and p53 codon 72 polymorphism on risk and age at onset of squamous cell carcinoma of the head and neck. Mol Carcinog 50: 697-706, 2011.
6. Markey MP: Regulation of MDM4. Front Biosci 16: 1144-1156, 2011

7. Yu H, Wang LE, Liu Z, Wei S, Li G, Sturgis EM and Wei Q Polymorphisms of MDM4 and risk of squamous cell carcinoma of the head and neck. Pharmacogenet Genomics 21: 388-396, 2011.

8. Kulkarni DA, Vazquez A, Haffty BG, Bandera EV,Hu W, Sun YY, Toppmeyer DL, Levine AJ and Hirshfield KM: A polymorphic variant in human MDM4 associates with accelerated age of onset of estrogen receptor negative breast cancer. Carcinogenesis 30: 1910-1915, 2009.

9. Fang S, Krahe R, Lozano G, Han Y, Chen W, Post SM, Zhang B, Wilson CD, Bachinski LL, Strong LC and Amos CI: Effects of MDM2, MDM4 and TP53 codon 72 polymorphisms on cancer risk in a cohort study of carriers of TP53 germline mutations. PLoS One 5: e10813, 2010.

10. Huang SF, Chen IH, Liao CT, Wang HM, Liou SH and Hsieh LL: Combined effects of MDM2 SNP 309 and p53 mutation on oral squamous cell carcinomas associated with areca quid chewing. Oral Oncol 45: 16-22, 2009.

11. Toledo F and Wahl GM: MDM2 and MDM4: p53 regulators as targets in anticancer therapy. Int J Biochem Cell Biol 39: 1476-1482, 2007.

12. Ménendez S, Goh AM, Camus S, Ng KW, Kua N, Badal V and Lane DP: MDM4 downregulates p53 transcriptional activity and response to stress during differentiation. Cell Cycle 10: 1100-1108, 2011.

13. Mancini F, Gentiletti F, D'Angelo M, Giglio S, Nanni S, D'Angelo C, Farsetti A, Citro G, Sacchi A, Pontecorvi A and Moretti F: MDM4 (MDMX) overexpression enhances stabilization of stress-induced p53 and promotes apoptosis. J Biol Chem 279: 8169-8180, 2004.

14. Atwal GS, Kirchhoff T, Bond EE, Montagna M, Menin C, Bertorelle R, Scaini MC, Bartel F, Bohnke A, Pempe C, et al: Altered tumor formation and evolutionary selection of genetic variants in the human MDM4 oncogene. Proc Natl Acad Sci USA 106: 10236-10241, 2009.

15. Nichols HB, Trentham-Dietz A, Hampton JM, Titus-Ernstoff L, Egan KM, Willett WC and Newcomb PA: From menarche to menopause: trends among US Women born from 1912 to 1969. Am J Epidemiol 164: 1003-1011, 2006.

16. Chang ET and Adami HO: The enigmatic epidemiology of nasopharyngeal carcinoma. Cancer Epidemiol Biomarkers Prev 15: 1765-1777, 2006

17. Tse KP, Su WH, Yang ML, Cheng HY, Tsang NM, Chang KP, Hao SP, Yao SY and Chang YS: A gender-specific association of CNV at 6 p21.3 with NPC susceptibility. Hum Mol Genet 20: 2889-2896, 2011.

18. Suda T, Yoshihara M, Nakamura Y, Sekiguchi H, Godai TI, Sugano N, Tsuchida K, Shiozawa M, Sakuma Y, Tsuchiya E, et al: Rare MDM4 gene amplification in colorectal cancer: The principle of a mutually exclusive relationship between MDM alteration and TP53 inactivation is not applicable. Oncol Rep 26: 49-54, 2011. 